Supporting Information

\title{
Recyclable Adsorbents based on Ceria \\ Nanostructures on Mesoporous Silica Beads for the Removal and Recovery of Phosphate from Eutrophic Waters
}

Ali Othman, Peter Vargo, and Silvana Andreescu*

* Department of Chemistry and Biomolecular Science, Clarkson University, Potsdam, New York 13699-5810, USA. E-mail: eandrees@clarkson.edu; Fax: +(315) 268 6610; Tel: +(315) 2682394 


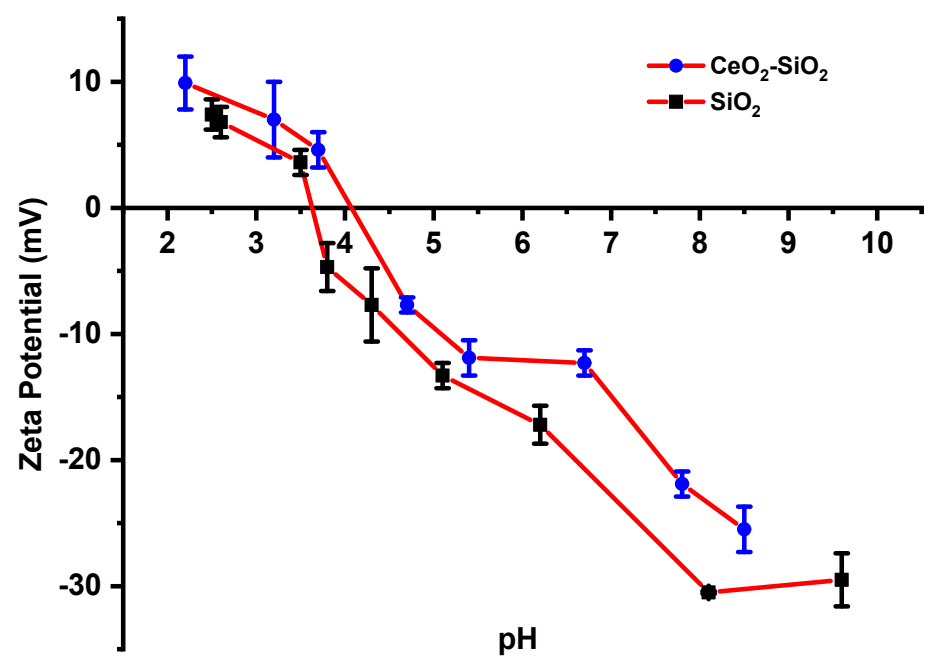

Figure S1. Zeta potential versus $\mathrm{pH}$ of $\mathrm{SiO}_{2}$ and $\mathrm{CeO}_{2}-\mathrm{SiO}_{2}$ adsorbent. 




Figure S2. Adsorption isotherms for surface area measurement of $\mathrm{SiO}_{2}$ and $\mathrm{CeO}_{2}-\mathrm{SiO}_{2}$ adsorbent by BET.

Table S1. The BET results of $\mathrm{SiO}_{2}$ beads and $\mathrm{CeO}_{2}-\mathrm{SiO}_{2}$ sorbent

\begin{tabular}{|l|l|l|l|}
\hline Sample & $\mathrm{S}_{\mathrm{BET}}\left(\mathrm{m}^{2} \mathrm{~g}^{-1}\right)$ & Pore volume $\left(\mathrm{cm}^{3} \mathrm{~g}^{-1}\right)$ & Pore width $(\mathrm{nm})$ \\
\hline $\mathrm{SiO}_{2}$ & 287.5 & 1.033 & 13.1 \\
\hline $\mathrm{CeO}_{2}-\mathrm{SiO}_{2}$ & 274.0 & 1.025 & 13.6 \\
\hline
\end{tabular}





Figure S3. ATR-FTIR of $\mathrm{SiO}_{2}$ and $\mathrm{CeO}_{2}-\mathrm{SiO}_{2}(\boldsymbol{A})$, and XPS survey spectra of (B) $\mathrm{SiO}_{2}$, inset: $\mathrm{Si}$ $2 p$ spectrum, and $(\boldsymbol{C}) \mathrm{CeO}_{2}-\mathrm{SiO}_{2}$, insets: $\mathrm{Ce} 3 \mathrm{~d}$ and $\mathrm{Si} 2 \mathrm{p}$ spectra. 


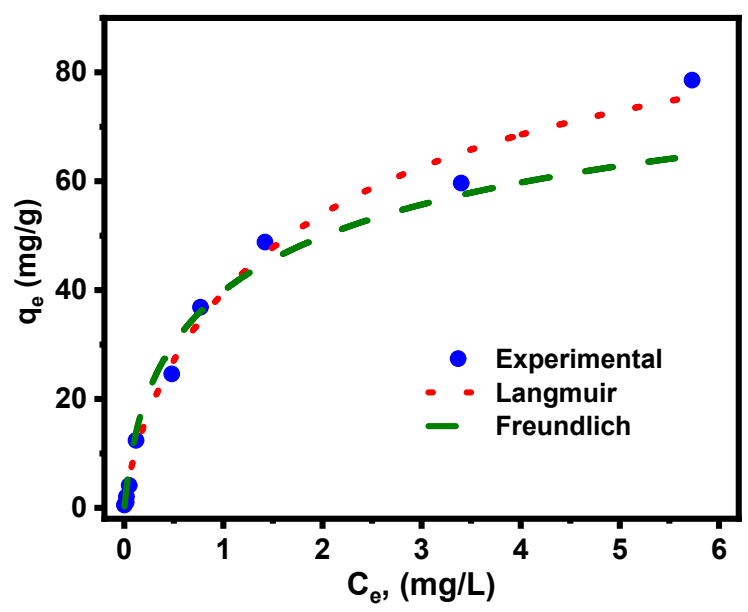

Figure S4. Adsorption equilibrium isotherm study of $\mathrm{CeO}_{2}-\mathrm{SiO}_{2}$ fitted with Langmuir and Freundlich isotherm models. (Adsorbent: $2 \mathrm{~g} / \mathrm{L} ;\left[\mathrm{PO}_{4}^{-3}\right]_{0}=0.5-100 \mathrm{mg} \cdot \mathrm{L}^{-1}$; time: $60 \mathrm{~min}$ ).

Table S2. Langmuir and Freundlich parameters of $\mathrm{PO}_{4}{ }^{3-}$ adsorption by $\mathrm{CeO}_{2}-\mathrm{SiO}_{2}$

\begin{tabular}{|c|c|c|c|c|c|}
\hline \multicolumn{3}{|c|}{ Langmuir } & \multicolumn{3}{|c|}{ Freundlich } \\
\hline $\begin{array}{l}q_{\max } \\
(m g / g)\end{array}$ & $\begin{array}{c}K_{L} \\
(L / m g)\end{array}$ & $R^{2}$ & $1 / n$ & $K_{f}$ & $R^{2}$ \\
\hline 110 & 0.56 & 0.991 & 0.36 & 39.8 & 0.963 \\
\hline
\end{tabular}




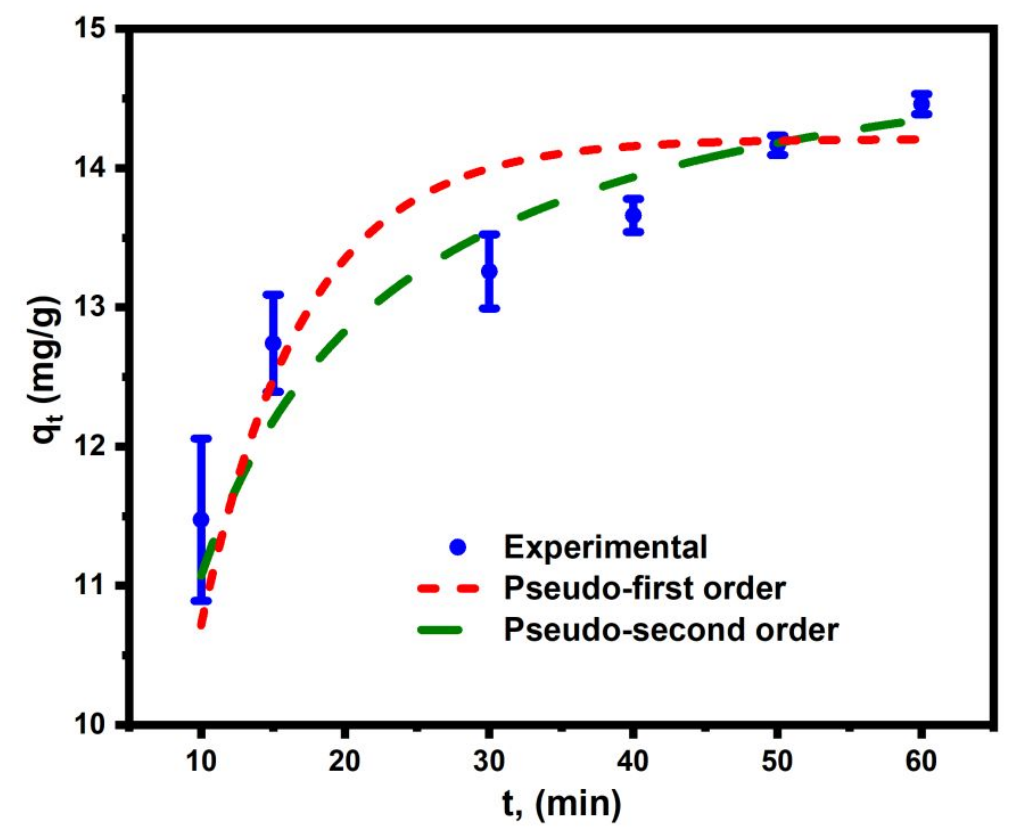

Figure S5. Pseudo-first and pseudo-second-order kinetics obtained by using the non-linear fitting

Table S3. Kinetic parameters of $\mathrm{PO}_{4}{ }^{3-}$ adsorption by $\mathrm{CeO}_{2}-\mathrm{SiO}_{2}$

\begin{tabular}{lll}
\hline Model & Parameters & Value \\
\hline Pseudo-first order & $q_{e}\left(m g \cdot g^{-1}\right)$ & 14.21 \\
& $K_{1}\left(\mathrm{~min}^{-1}\right)$ & 0.140 \\
& $R^{2}$ & 0.524 \\
Pseudo-second order & $q_{e}\left(m g \cdot g^{-1}\right)$ & 15.25 \\
& $K_{2}\left(g \cdot m g^{-1} \cdot \mathrm{min}^{-1}\right)$ & 0.017 \\
& $R^{2}$ & 0.857 \\
\hline
\end{tabular}


Table S4. Z-potential measurement of the regeneration process

\begin{tabular}{|l|l|}
\hline Sample & $\begin{array}{l}\text { Z-potential } \\
(\mathrm{mV})\end{array}$ \\
\hline $\mathrm{CeO}_{2}-\mathrm{SiO}_{2}$ & $-7.0 \pm 1.9$ \\
\hline $\mathrm{CeO}_{2}-\mathrm{SiO}_{2} / \mathrm{H}_{2} \mathrm{O}_{2}$ & $-18.0 \pm 1.7$ \\
\hline $\mathrm{CeO}_{2}-\mathrm{SiO}_{2} / \mathrm{PO}_{4}{ }^{3-}$ & $-11.2 \pm 1.8$ \\
\hline $\mathrm{CeO}_{2}-\mathrm{SiO}_{2} / \mathrm{PO}_{4}{ }^{3-} / \mathrm{H}_{2} \mathrm{O}_{2}$ & $-13.1 \pm 2.1$ \\
\hline
\end{tabular}




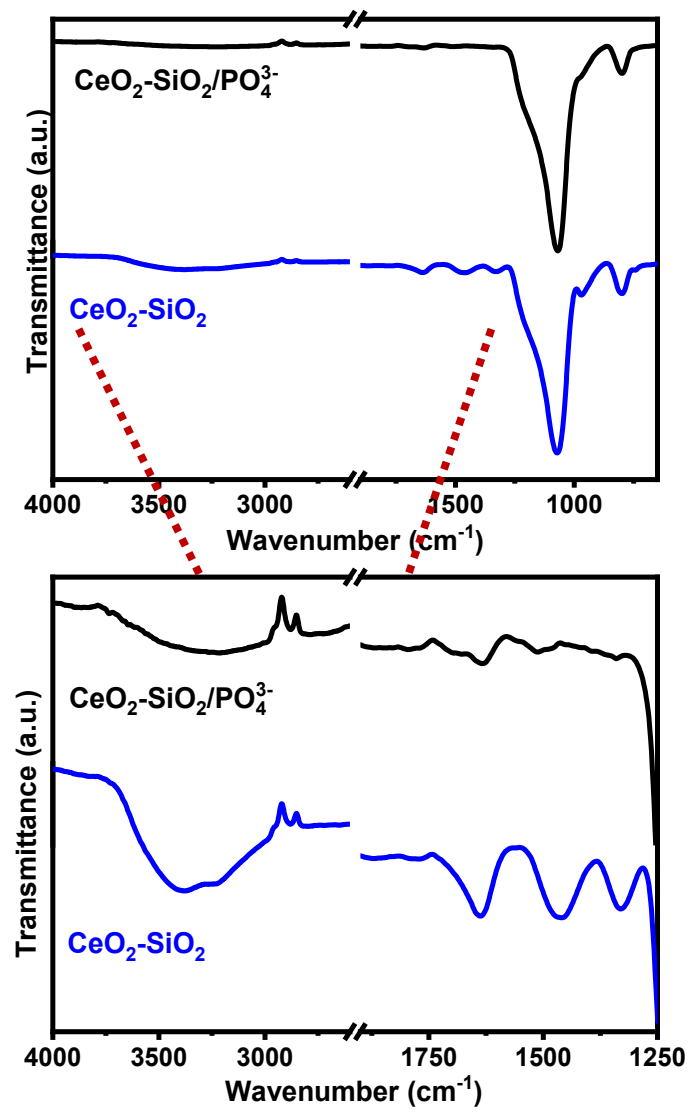

Figure S6. ATR-FTIR spectra of $\mathrm{CeO}_{2}-\mathrm{SiO}_{2}$ and $\mathrm{CeO}_{2}-\mathrm{SiO}_{2} / \mathrm{PO}_{4}{ }^{3-}$. 